\title{
A Fractal Menger Sponge Space-Time Proposal to Reconcile Measurements and Theoretical Predictions of Cosmic Dark Energy
}

\author{
Mohamed S. El Naschie \\ Department of Physics, University of Alexandria, Egypt \\ Email: Chaossf@aol.com
}

Received February 12, 2013; revised March 12, 2013; accepted March 31, 2013

Copyright (C) 2013 Mohamed S. El Naschie. This is an open access article distributed under the Creative Commons Attribution License, which permits unrestricted use, distribution, and reproduction in any medium, provided the original work is properly cited.

\begin{abstract}
The 95.5 percent of discrepancy between theoretical prediction based on Einstein's theory of relativity and the accurate cosmological measurement of WMAP and various supernova analyses is resolved classically using Newtonian mechanics in conjunction with a fractal Menger sponge space proposal. The new energy equation is thus based on the familiar kinetic energy of Newtonian mechanics scaled classically by a ratio relating our familiar three dimensional space homology to that of a Menger sponge. The remarkable final result is an energy equation identical to that of Einstein's $E=m c^{2}$ but divided by 22 so that our new equation reads as $E=\frac{m c^{2}}{22}$. Consequently the energy Lorentz-like reduction factor of $\gamma=\frac{1}{22} \cong 4.5$ percent is in astonishing agreement with cosmological measurements which put the hypothetical dark energy including dark matter at $[1-(1 / 22)](100) \cong 95.5$ percent of the total theoretical value. In other words our analysis confirms the cosmological data putting the total value of measured ordinary matter and ordinary energy of the entire universe at 4.5 percent. Thus ordinary positive energy which can be measured using conventional methods is the energy of the quantum particle modeled by the Zero set in five dimensions. Dark energy on the other hand is the absolute value of the negative energy of the quantum Schrödinger wave modeled by the empty set also in five dimensions.
\end{abstract}

Keywords: Menger Sponge Space; Revising Relativity; Dark Energy; Energy of the Quantum Particle; Energy of the Quantum Wave; Kähler Manifold as Space-Time; Modified Lorentz Transformation

\section{Introduction}

The discrepancy between theoretical prediction and cosmological measurements of the entire energy content of our universe [1-3] is resolved in the present work. This is achieved by combining classical Newtonian mechanics with a novel fractal interpretation of our familiar classical space. We start by assuming that space itself is a Cantorian set-like fractal akin to a Menger sponge $[4,5]$. This immediately leads us to qualitative and equally important, if not more important, quantitative results [6]. From the topology and geometry of the Menger sponge $[4,5,7]$ and the classical expression for kinetic energy we can draw the inference that only $4.5 \%$ of the entire energy of the cosmos is ordinary matter and energy $[1,3,6]$. The rest of what Einstein's equation predicted, namely
$100 \%-4.5 \%=95.5 \%$ is actually due to the zero fractal volume of the Menger sponge-like "non-space" (see Figures 1 and 2) which exists indirectly by not being there or being there only in the Aristotelian sense of Potentia not unlike many other things in quantum mechanics such as the well documented Bohm-Aharonov effect [4-6]. The matter and energy corresponding to this space structure with a relatively large Hausdorff dimension but a zero classical 3D volume, if they can be called matter and energy in the ordinary sense at all, are for the time being and the foreseeable future completely inaccessible to us [1-3]. The situation is not dissimilar to the zero and empty set of transfinite set theory because zero and negative Menger-Urysohn dimensions [6,7], although referring to zero and empty sets, are still indispensible to a logical, coherent, complete and consistent set theory and 


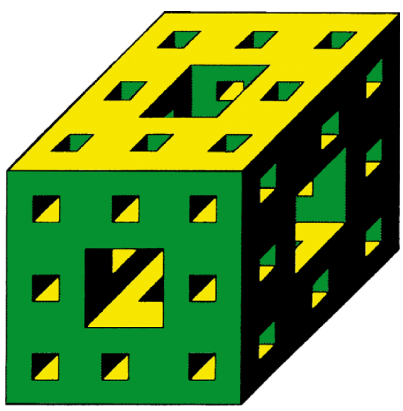

(a)

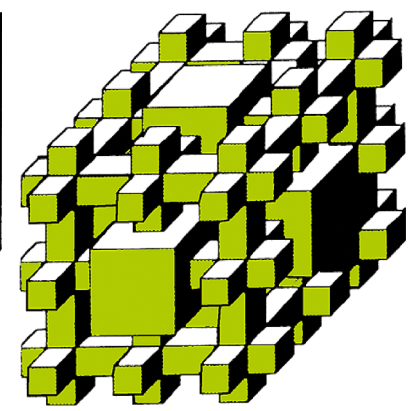

(b)

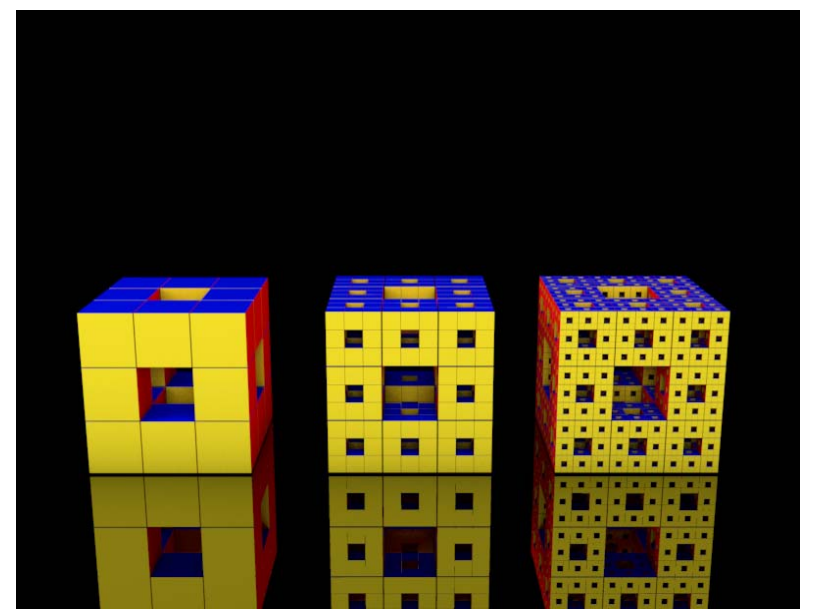

(c)

Figure 1. (a) The Menger sponge. The Hausdorff dimension is given by $D_{M S}=\frac{\ln 20}{\ln 3}=2.7268$. The microwave background temperature is found to be $T_{C}(\mathrm{COBE})=D_{\mathrm{MS}} K=$ $2.726 \mathrm{~K}$; (b) The complement of a Menger sponge. The Hausdorff dimension is $D_{C M S}=3-D_{M S}=\left(\frac{\ln 20}{\ln 3}\right)$. That means $D_{C M S}=3-D_{M S}=3-2.726833=0.27316$. One of the first proposals of a Menger sponge-like universe came from J. Syldovych based probably upon an even earlier conjecture by the Swedish astronomer C. Charlier; (c) Various iteration stages leading to a Menger sponge in the infinite limit which is beyond graphic representation.

thus mathematics and consequently physics $[7,8]$.

The present analysis starts by showing that Einstein's $E=m c^{2}[9,10]$ must be revised to $E=\gamma m c^{2}=(1 / 22) m c^{2}$ and conclude that

$$
\gamma=\frac{E(\text { Einstein })}{E(\text { quantum relativity })}=\frac{1}{22} \cong 4.5 \%
$$

in complete agreement with the WMAP and supernova measurements [1-3]. This means that only $4.5 \%$ of the expected energy exists while the rest of $95.5 \%$ must be assumed to be missing and is therefore referred to as "dark" or missing energy [1-3,6]. Subsequently the analysis is refined and extended to find the exact $\gamma$ which turned out to be $1 / 22.18033989$ being the ratio of

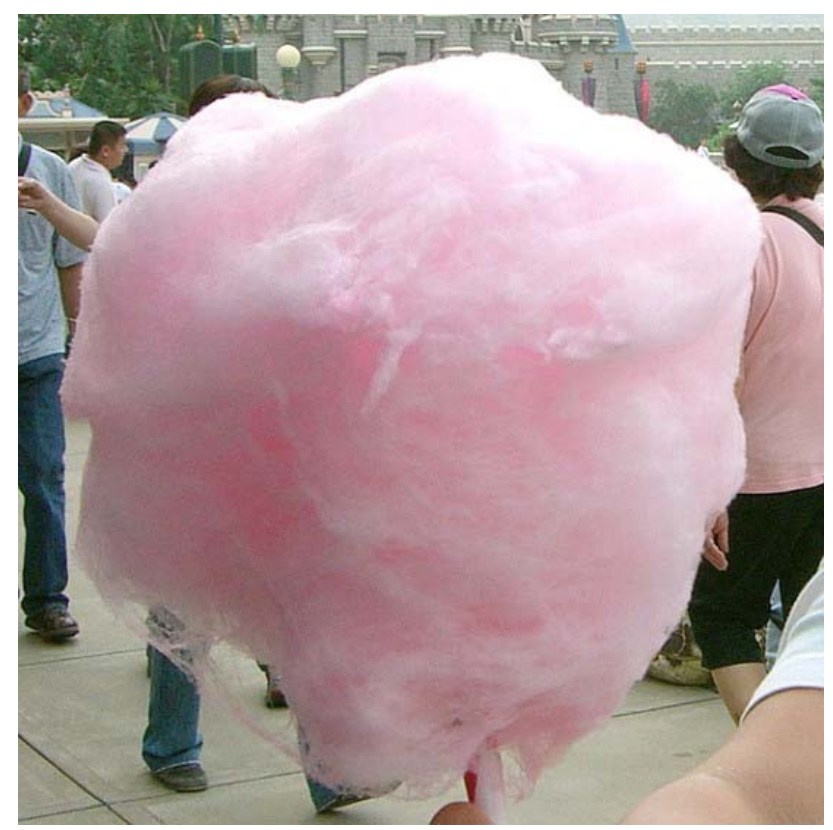

Figure 2. The entire cosmos as a cotton candy. This would be a possibility for explaining how such a huge form is only $4.5 \%$ of what it seems to be.

$D^{(4)}=4$ and $D_{F}^{(4)}=4-k=4-\phi^{3}\left(1-\phi^{3}\right)$ where $\phi=\frac{\sqrt{5}-1}{2}$. Consequently the exact $\gamma$ is given by

$$
\begin{aligned}
\gamma_{Q G} & =\frac{\left(D^{(4)}-D_{F}^{(4)}\right)}{D^{(4)}}=\frac{(4-(4-k))}{4} \\
& =\frac{\phi^{5}}{2}=\frac{1}{22.18033989} \square \frac{1}{22}
\end{aligned}
$$

It should be noted that $\phi^{5}$ is the well known Hardy's probability of quantum entanglement [11]. This fact reveals the quantum roots of our classical theory and we note on passing that dark energy is the absolute value of the negative energy of the quantum Schrödinger wave while the positive ordinary energy is that of the quantum particle, a subject which will not be discussed within the present work but is explained in some detail in Overview Charts No. 1-3 as well as Figures 3 and 4 [8,11].

\section{Analysis}

\subsection{Classical Analysis Using the Menger Sponge}

A Menger sponge is basically a three dimensional fractal $[4,5,7]$ constructed by drilling infinitely many cubic holes into it iteratively, the result of which is shown in Figure 1(c). A discussion of this well known fractal with numerous applications in physics, chemistry and biology may be found in many of the excellent text books on the subject $[4,5,7]$. Assuming empty space itself and not 


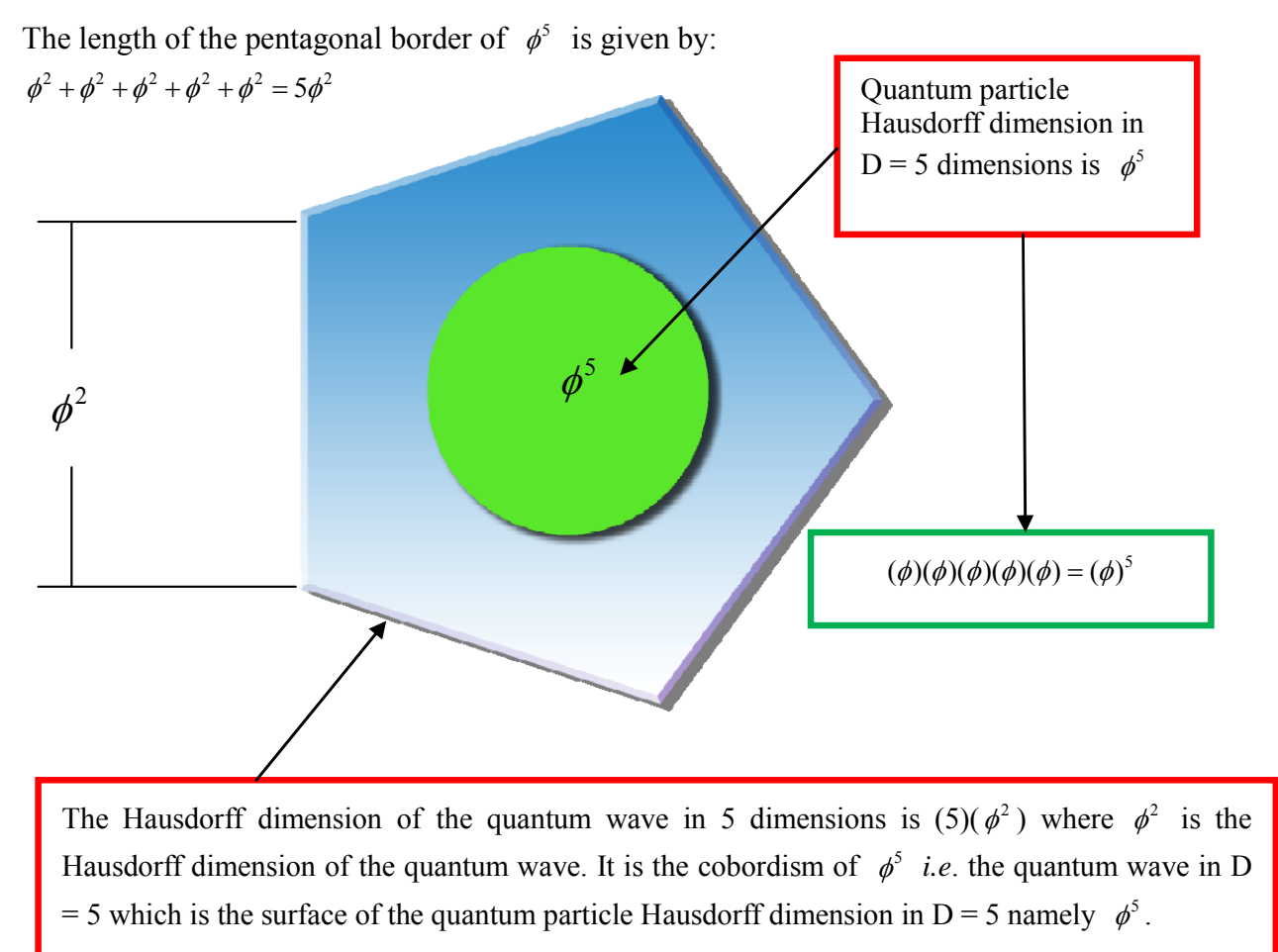

The length of the circumference of the above pentagon is $(5)\left(\phi^{2}\right)$ where $\phi^{2}$ is the length of each side and $\phi=2 /(1+\sqrt{5})$. Note that $\phi^{2}$ is the Hausdorff dimension of the wave i.e. the surface of the particle given by the Hausdorff dimension $\phi$. Thus the length of circumference of the pentagon $5 \phi^{2}$ maybe thought of naively as the surface area of the volume $\phi^{5}$ inside $5 \phi^{2}$. Thus $5 \phi^{5}$ decides on the dark energy of the quantum wave. Note that the set theoretical operation corresponding to $5 \phi^{2}$ is union of sets while in theory of probability, it is the addition theorem. Finally we should mention that the largest volume of a sphere exits in five K-K i.e. Kaluza- Klein dimensional space. All other smaller or larger dimensions possess a smaller sphere volume. Adding that a seven dimensional sphere possesses the largest surface area we see that $(5+7)=12$ is an optimal dimension for spacetime with two time dimensions like in F Theory. Restricting to one time dimension we find Witten's eleven dimensional theory $(12-1=11)$.

Figure 3. A naive geometrical interpretation of geometrical density or Hausdorff measure $\operatorname{vol}\left(D_{-1}\right)=5 \phi^{2}$ which decides upon the magnitude of dark energy density of the quantum wave $E($ Dark $)=\frac{1}{2}\left(5 \phi^{2}\right) m c^{2}$.

merely matter to be a Menger sponge fractal, then the Hausdorff dimension of this space could be set equal to the Menger sponge (see Figure 1(a)):

$$
D_{H}(M)=\frac{\ln 20}{\ln 3}=2.72683302 .
$$

Let us now ponder carefully what $D_{H}(M)$ really measures and refers to. Since the original cube was obviously 3 dimensions and we have at least in theory removed almost the entire substance, i.e. space which makes it up, then it follows that the large dimension of $\frac{\ln 20}{\ln 3}$ refers basically the quasi-Hausdorff value to the space removed rather than the sparse Cantor point set left. Said in a different way the volume of the Menger sponge space is now zero and nothing is left except a zero measure infinitely long and infinitely thin fractal line in three dimensional classical spaces. What could be said to have remained from this $3 \mathrm{D}$ space is a zero volume Menger fractal of a Hausdorff dimension equal to that of the complement space of the Menger sponge and given by (see Figure 1(b))

$$
D^{C}=D^{(3)}-D_{H}(M)=3-\frac{\ln 20}{\ln 3}=0.2731669721 .
$$




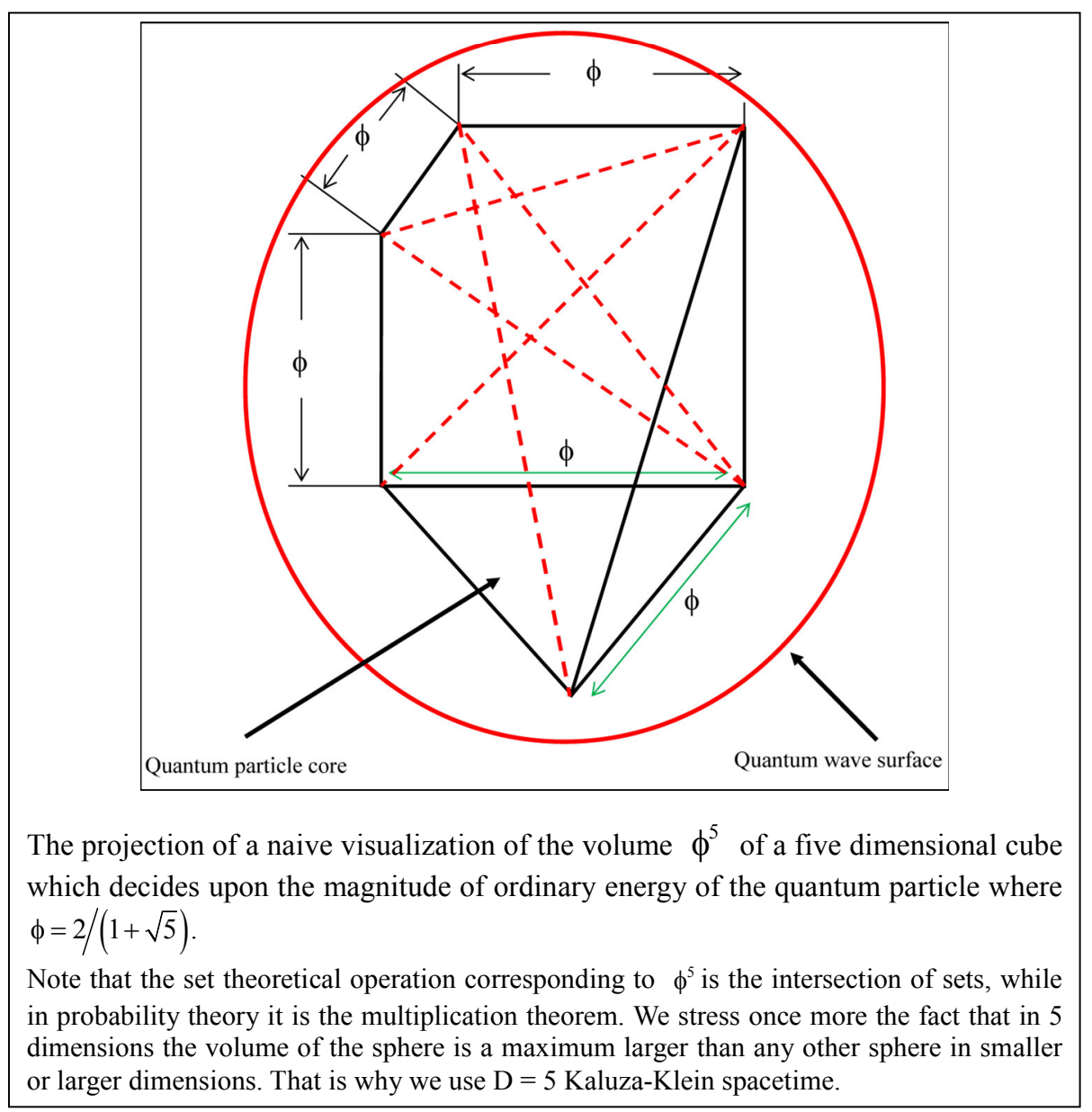

Figure 4. A naive geometrical interpretation of $\operatorname{vol}\left(D_{0}\right)=5 \phi^{5}$ of the ordinary energy density of the quantum particle $E($ ordinary $)=\frac{1}{2}\left(\phi^{5}\right) m c^{2}$.

It is important to realize that the relative ratio of what is left of real space to the original $3 \mathrm{D}$ cube is obviously the difference between 3D "solid" and "smooth" Euclidean space and a cotton candy-like (see Figure 2) Menger sponge dimension divided by 3D. In other words our space "density" ratio is

$$
\begin{aligned}
\gamma & =\frac{D^{(3)}-D_{H}(M)}{D^{(3)}}=\frac{3-(\ln 20 / \ln 3)}{3} \\
& =0.09105565738 .
\end{aligned}
$$

It is thus imperative to understand that this $\gamma$ must be included in the classical kinetic energy expression of Newton which presupposes a "smooth" "solid" nonfractal space. Consequently

$$
E=(1 / 2) m v^{2}
$$

must be logically extended to

$$
E=\frac{\gamma}{2} m(v \rightarrow c)^{2}
$$

and therefore

$$
E=\left(\frac{0.0910556573}{2}\right) m c^{2}
$$

That means

$$
E_{Q R}=\frac{m c^{2}}{21.96458801} \square \frac{m c^{2}}{22} .
$$

This is only $4.5 \%$ from what the relativistic nonquantum equation of Einstein predicts. However it is clear from the full agreement of the energy predicted by $E_{Q R}$ with the accurate experimental measurement of WMAP and others [1-3] that $E=m c^{2}$ does not apply to extreme situations like when considering the cosmos as a whole.

In the next section we will give some deeper and mathematically more sophisticated reasons why $E_{Q R}$ is the correct equation for calculating the energy of the cosmos and that $\gamma=\frac{1}{22}$ could be seen as resulting from 
accounting for a fundamental quantum mechanical effect, namely quantum entanglement $[8,11]$.

\subsection{Quantum Relativity Analysis}

It is well known that Hardy's quantum probability $[8,11]$ is generic and is given by

$$
P(\text { Hardy })=\phi^{5}
$$

where $\phi=\frac{2}{(1+\sqrt{5})}[8,11]$. At least in theory the two particles $P=\phi^{5}$ which were tested to very high accuracy experimentally lead to the conclusion that for a single particle we would have

$$
P(\text { Hardy for one particle })=\frac{\phi^{5}}{2} .
$$

Now Einstein's equation is a one particle equation

$$
E=m c^{2} \text {. }
$$

Intersecting this relativistic formula with the quantum formula, a quantum relativistic energy formula is easily found to be (see Figures 5 and $\mathbf{6}$ )

$$
E_{Q R}=\frac{\phi^{5}}{2} m c^{2}=\frac{m c^{2}}{22.18033989} \cong \frac{m c^{2}}{22} .
$$

This is almost the same result obtained earlier on using classical mechanics and the Menger sponge space in the previous Section 2.1.

\subsection{Analysis Using K3 Kähler Manifold}

The Kähler manifolds are used for compactification in superstrings and related theories [8]. Let us assume that

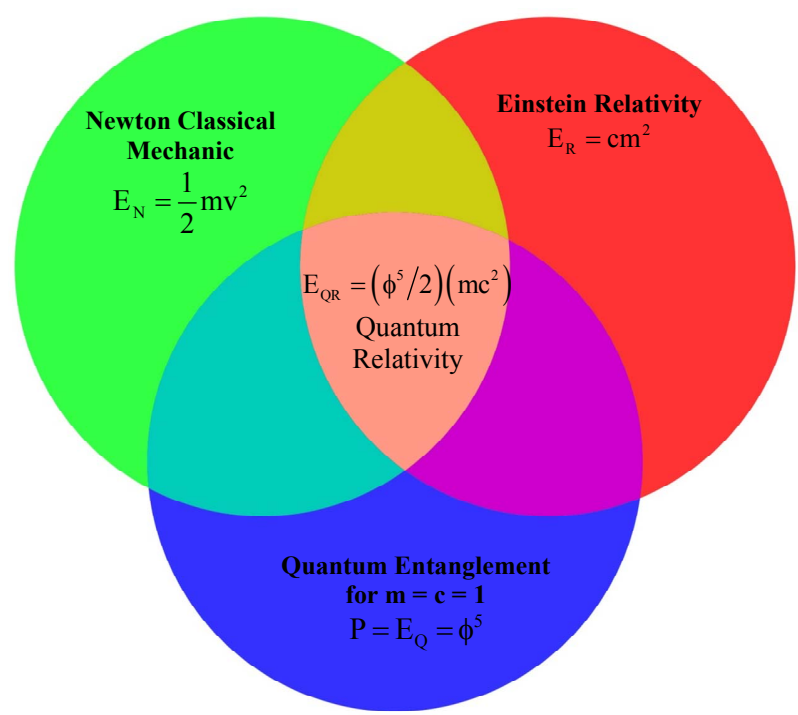

Figure 5. Quantum relativity theory as an intersection of the three major fundamental theories of physics.

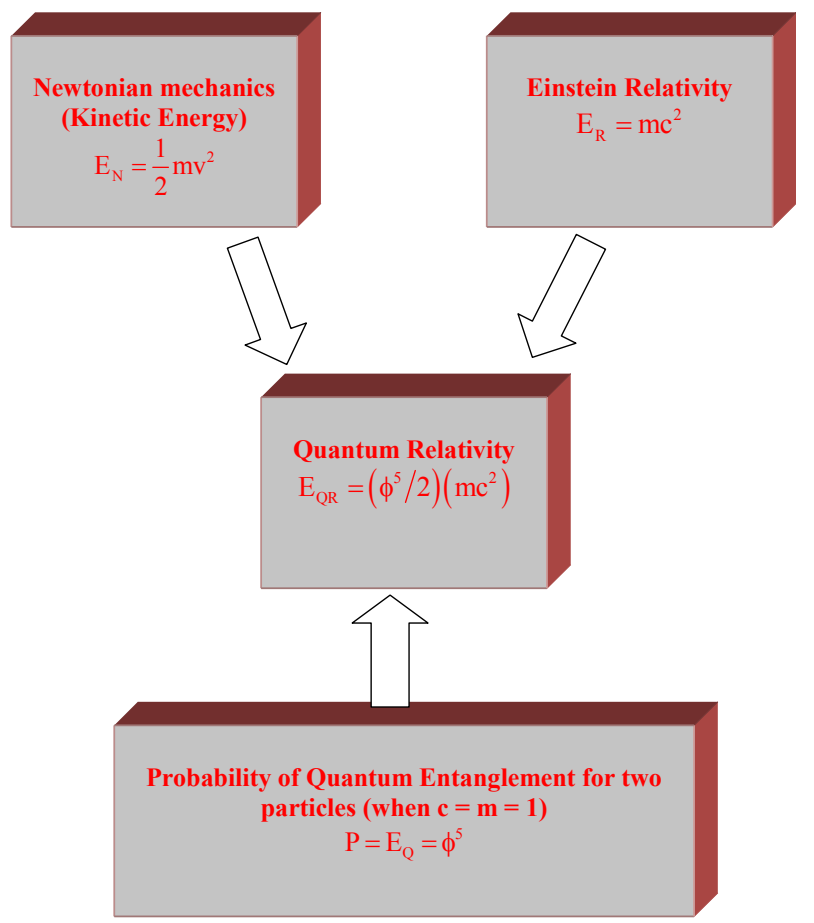

Figure 6. The effective quantum gravity energy formula $E_{Q R}=\left(\phi^{5} / 2\right)\left(m c^{2}\right)$ as a synthesis of Newton, Einstein and quantum theories.

space and time are fused together and modeled by such a Kähler manifold. The Betti number $b_{2}$ for K 3 is given by $[12,13]$

$$
b_{2}=19+3=22
$$

This number could be thought of as counting the number of 3D holes in K3. Thus compared with Einstein's 4D smooth manifold for which $b_{2}=1$, our K3 has 22 times more $3 \mathrm{D}$ holes in it $[12,13]$. Thus we could write the ratio $\lambda$ as $[12,13]$

$$
\lambda=\frac{b_{2}(\text { Einstein })}{b_{2}(\text { K3 Kähler })}=\frac{1}{22} .
$$

This is obviously a very useful scaling exponent and we see that $\lambda=\gamma$ and consequently multiplied with $E=m c^{2}$ of Einstein we find again our $E_{Q R}$ energy formula

$$
E=(\lambda=\gamma)\left(m c^{2}\right)=\frac{m c^{2}}{22}
$$

Thinking deeply about this result one may be yet again surprised to realize that in retrospect, it should have been expected for the following obvious reason. The difference between Newton's kinetic energy formula $E=(1 / 2) m v^{2}$ and Einstein's maximal energy $E=m c^{2}$ is formally a factor half and setting $v=c$. Subsequently we showed that $E_{Q R}=\frac{m c^{2}}{22}$ by assuming a different 
Menger fractal geometry instead of the smooth geometry of Newton's space. Here again $E_{Q R}$ kept the same form of Newton and Einstein and everything else was taken care of by a simple factor $1 / 22$. Then in our second derivation the same result was found after fusing quantum entanglement with special relativity. Again if we remember that gauge theory started with the idea of Weyl scaling and that Nottale's high energy particle physics and cosmology theory is based on scale relativity principle, then we realize that this was also to be expected in our case. For these reasons the ratio of the homology of a classical geometry such as $b_{2}=1$ of Einstein's space and the $b_{2}=$ 22 of a complex manifold like our K3 used here [12,13] harbors more than meets the eyes in the harmless appearance of a simple scaling factor.

\subsection{The Lorentz-Like Transformation Leading to Quantum Relativity}

To connect all the preceding three different derivations with the original theory of Lorentz and Einstein, it is instructive to see that a similar derivation in the spirit of Lorentz-Einstein transformation holds and leads to the same result of quantum relativity $E_{Q R} \cong \frac{m c^{2}}{22}$. Accepting the three fundamental phenomenological effects of special relativity, the following transformations are evidently consistent, i.e. [7]

$$
\begin{aligned}
& m(\text { mass }) \rightarrow m(1+\beta) \\
& x(\text { space coordinate }) \rightarrow x(1-\beta) \\
& t(\text { time }) \rightarrow t(1+\beta)
\end{aligned}
$$

where $\beta$ is a boost which does not need to be defined by anything related directly to $v / c$ where $c$ is the phenomenologically and experimentally accepted constant speed of light. Inserting in Newton's kinetic energy we find

$$
E_{1}=\frac{1}{2}\left(\frac{1-\beta}{1+\beta}\right)^{2}(1+\beta) m(v \rightarrow c)^{2} .
$$

On the other hand we could use the conventional Lorentz transformation in the unconventional form of light cone velocity used in superstrings quantization $[14,15]$ and extend it to encompass a light cone mass as follows:

$$
v \rightarrow \frac{1-\beta}{1+\beta}, m \rightarrow \frac{1}{\sqrt{1-\beta}} .
$$

Inserting again the Newton kinetic energy we find

$$
E_{2}=\frac{1}{2}\left(\frac{1-\beta}{1+\beta}\right)^{2} \frac{1}{\sqrt{1-\beta}} .
$$

Setting $v=c, c=m=1$ and equating $E_{1}$ and $E_{2}$ one finds

$$
\frac{1}{2}\left(\frac{1-\beta}{1+\beta}\right)^{2}(1+\beta)=\frac{1}{2}\left(\frac{1-\beta}{1+\beta}\right)^{2} \frac{1}{\sqrt{1-\beta}} .
$$

This leads to a quadratic equation in $\beta$ with the only positive root $\beta=\phi$ [16]. Inserting in $E_{1}$ one finds immediately that

$$
E_{1}=E_{Q R}=\frac{\phi^{5}}{2} \cong \frac{m c^{2}}{22}
$$

which confirms without any doubt the correctness of all the previous three derivations of Sections 2.1, 2.2 and 2.3 of the present work. In Chart Nos. 4 and 5 we give an overview comparing different Lorentz-like transformations leading to the same robust result $E_{Q R} \cong \frac{m c^{2}}{22}$.

\section{Negative Gravity as Compactified Dimensions}

When an elastic surface is acted upon with a load, it curves $[17,18]$. The theory of such elastic surfaces is highly developed in a remarkably successful theory called theory of elasticity [17-20]. This theory and its sister, theory of plasticity, is the basis of all structural engineering science which gave us shell structures [17-19] covering large sports and airport halls without supporting columns and thin fuselages which carry passengers across the Atlantic in a few hours. When such an elastic or elasto-plastic surface is sufficiently thick, long and narrow then an interesting curvature phenomena takes place called anticlastic curvature [20]. The point is that when the long thick elastic structure is bent, then its cross section curves in the opposite direction. This classical analogy is helpful to visualize the effect of the compactified 22 dimensions belonging to the 26 dimensions of say the heterotic superstring theory or the old bosonic string theory of Veneziano and Nambu [14]. Thus we are suggesting here that $26-4=22$ compactified dimensions are a string analogy to the anticlastic curvature observed in thick elastic structural beams as well as long, thin walled elastic tubes subjected to local singular loads somewhere in the middle of the length direction [18]. In turn this anticlastic curvature and the corresponding compactified 22 dimensions produce the effect of negative gravity which can explain the observed increased acceleration in the expansion of the universe $[1-3,6]$. Figure 7 and Chart No. 6 may help in understanding the basic idea behind negative gravity. Thus we advocate that the 22 compactified, curled extra dimensions are not only the cause of dark energy, but that they also play the role of Einstein's cosmological constant or negative gravity. Similar qualitative conclusions may be drawn using the theory of polar media due to the brothers 


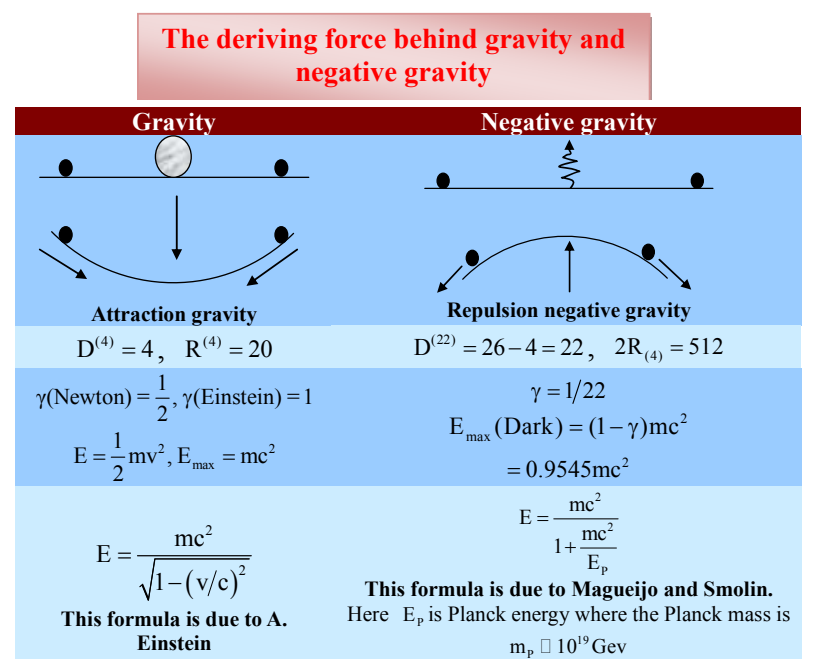

Figure 7. Here $D^{(4)}=4$ is the dimension of Einstein's spacetime, $R^{(4)}=20$ are the number of independent components of Riemann curvature tensor in dour dimensions and $R_{(4)}=$ 256 are the number of all the components of the same curvature tensor, $v$ is the velocity and $c$ is the speed of light.

Cosserat [19] and also using Cartan's torsional curvature $[9,10,17]$.

\section{The Role of Transfinite and Hyperbolic Geometry}

The thread connecting the different themes of all the preceding sections is the profound impact of non-classical and hyperbolic geometry on physics. In this section we stress this point by referring to the explicit impact of non-classical geometry and its Lie symmetry groups as presented in overview Chart 7 on physics [12-16].

\section{Conclusions}

Assuming that space-time is akin to a Menger sponge fractal we were able to show that a purely classical energy expression $E=(1 / 2) m v^{2}$ changes to

$$
E=\frac{1}{22} m(v \rightarrow c)^{2}
$$

The result of this Newtonian non-relativistic and nonquantum derivation is confirmed using a variety of sophisticated mathematical methods including a Lorentzlike transformation as well as an intersection between Hardy's quantum entanglement

$$
P(H)=\phi^{5}=\frac{1}{22.18033989}
$$

and Einstein's maximal energy $E=m c^{2}$. Thus $E_{Q R}=\frac{\phi^{5}}{2} m c^{2}$ may be regarded as a quantum relativity formula and therefore $\gamma=\frac{\phi^{5}}{2} \cong \frac{1}{22}$ may be viewed in various ways as:

1) A Weyl-Nottale scaling expression for quantum relativity [6].

2) A measure for the hypothetical dark energy of the cosmos

$$
[1-(1 / 22)](100) \cong 95.5 \%
$$

in full agreement with measurements [1-3,6].

2) The magnitude of quantum entanglement involved in quantum relativity at the Hubble radius scale of the universe [6].

3) A measure for the negative gravity or anticlastic curvature effect responsible for the increasing rate of expansion of the universe.

4) The ratio of two Betti numbers characterizing the homology of Einstein's space and a K3 Kähler space namely $\lambda=\frac{\left.b_{2} \text { (Einstein }\right)}{b_{2}(\mathrm{~K} 3 \mathrm{Kähler})}=\frac{1}{22} \quad[12,13]$.

It is important to note that recent investigation by the present author has revealed that $E \cong \frac{m c^{2}}{22}$ is the energy of the quantum particle while $E \cong m c^{2} \frac{21}{22}$ is the dark energy of the quantum wave. The sum is Einstein's energy $E=m c^{2}$. Thus Einstein's formula is blind to any distinction between ordinary energy and dark energy. (See also Overview Charts 1-3 and Figures 6 and 7).

\section{REFERENCES}

[1] E. J. Copeland, M. Sami and S. Tsujikawa, "Dynamics of Dark Energy," 2006.

[2] L. Amendola and S. Tsujikawa, "Dark Energy: Theory and Observations," Cambridge University Press, Cambridge, 2010. doi:10.1017/CBO9780511750823

[3] S. Perlmutter, et al., "Supernova Cosmology Project Collaboration. Measurements of Omega and Lambda from 42 High-Redshift Supernova," The Astrophysical Journal, Vol. 517, 1999, pp. 565-585. doi: $10.1086 / 307221$

[4] B. Mandelbrot, "The Fractal Geometry of Nature," Freeman, San Francisco, 1982.

[5] M. S. El Naschie, "Elementary Prerequisites for E-Infinity," Chaos, Solitons \& Fractals, Vol. 30, No. 3, 2006, pp. 579-605. doi:10.1016/j.chaos.2006.03.030

[6] M. S. El Naschie and L. Marek-Crnjac, "Deriving the Exact Percentage of Dark Energy Using a Transfinite Version of Nottale's Scale Relativity," International Journal of Modern Nonlinear Theory and Application, Vol. 1, No. 4, 2012, pp. 118-124. doi:10.4236/ijmnta.2012.14018

[7] M. S. El Naschie, "A Review of E-Infinity and the Mass spectrum of high energy particle physics," Chaos, Solitons \& Fractals, Vol. 19, No. 1, 2004, pp. 209-236. 


\section{doi:10.1016/S0960-0779(03)00278-9}

[8] M. S. El Naschie, "Quantum entanglement as a Consequence of a Cantorian Micro Space-Time Geometry," Journal of Quantum Information Science, Vol. 1, No. 2, 2011, pp. 50-53. doi:10.4236/jqis.2011.12007

[9] W. Rindler, "Relativity, Special, General and Cosmological," Oxford Press, Oxford, 2004.

[10] J.-P. Hsu and L. Hsu, "A Broad View of Relativity," World Scientific, Singapore, 2006.

[11] L. Hardy, "Non-Locality of Two Particles without Inequalities for Almost All Entangled States," Physical Review Letters, Vol. 71, No. 11, 1993, pp. 1665-1668. doi:10.1103/hysRevLett.71.1665

[12] D. Joyce, "Compact Manifold with Special Holonomy," Oxford Press, Oxford, 2003.

[13] M. S. El Naschie, “On Two New Fuzzy Kähler Manifolds, Klein Modular Space and 't Hooft's Holographic Principles," Chaos, Solitons \& Fractals, Vol. 29, No. 4, 2006, pp. 876-881. doi:10.1016/j.chaos.2005.12.027
[14] J. Polchinski, "String Theory," Cambridge University Press, Cambridge, 1998.

[15] M. Duff, "The World in Eleven Dimensions," IOP Publishing, Bristol, 1999.

[16] S. Hendi and M. Sharifzadeh, "Special Relativity and the Golden Mean," International Journal of Theoretical Physics, Vol. 1, No.1, 2012, pp. 37-45.

[17] W. Koiter, "Elastic Stability and Post Buckling Behavior in Nonlinear Problems," University of Wisconsin Press, Madison, 1963, pp. 257-275.

[18] M. S. El Naschie, "Stress, Stability and Chaos in Structural Engineering," McGraw Hill, London, 1990.

[19] E. Cosserat and F. Cosserat, "Theorie des Corps Deformable," Paris, 1909.

[20] D. Horrocks and W. Johnson, "On Anticlastic Curvature with Special Reference to Plastic Bending," International Journal of Mechanical Sciences, Vol. 9, No 12, 1967, pp. 835-861. doi:10.1016/0020-7403(67)90011-2 


\section{Overview Chart}

Ordinary positive energy of the quantum particle and negative dark energy of the quantum wave from the zero set and the empty set respectively via Kaluza-Klein $\mathrm{D}=5$ spacetime $[42,43]$.

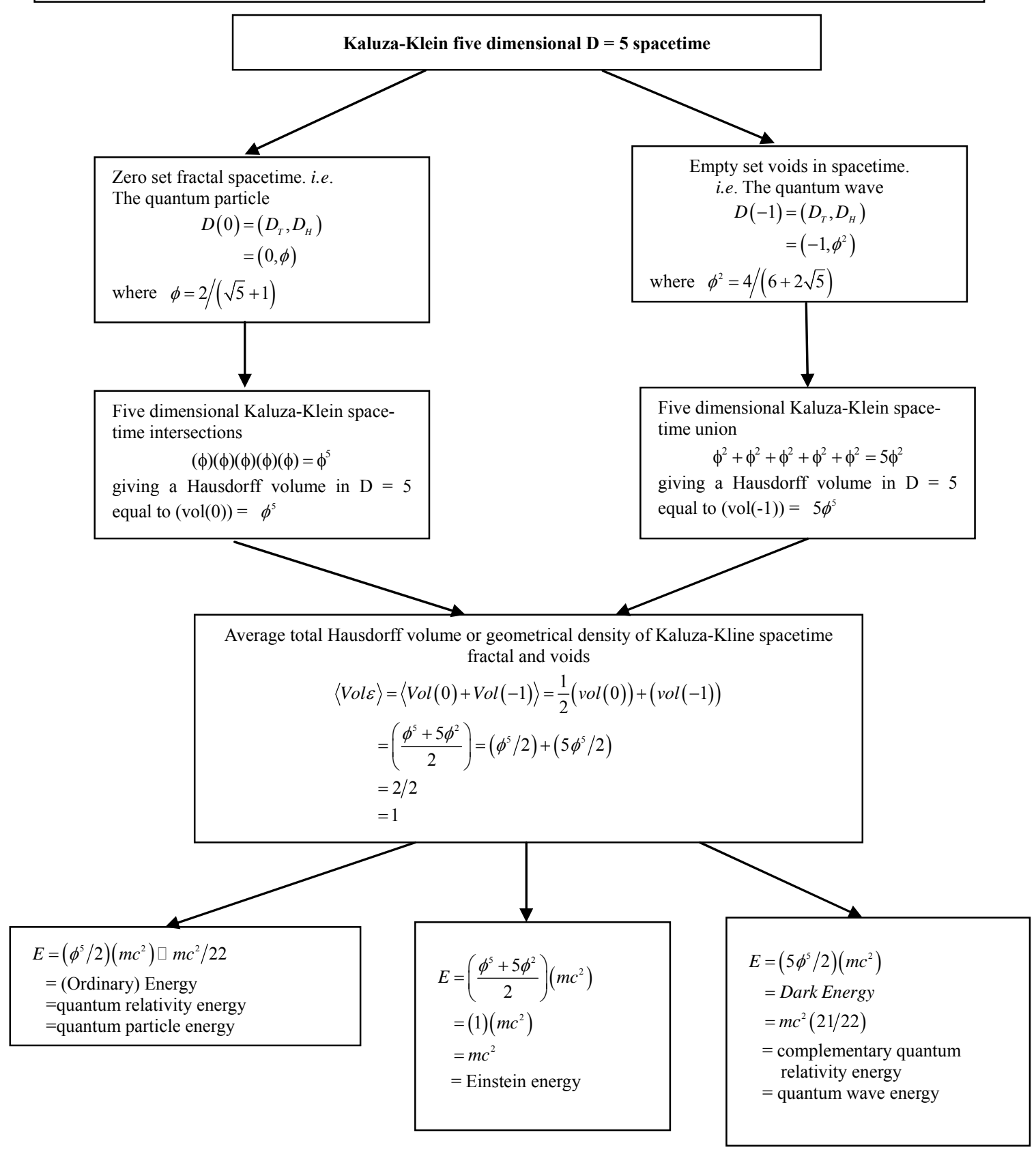

\footnotetext{
Note that in all above explanations we let the velocity $\mathrm{v}$ tend simply to that of the velocity of light $(\mathrm{v} \rightarrow \mathrm{c})$ and the division by 2 is due to fractal averaging and not due to using Newton's kinetic energy $E_{N}=\frac{1}{2} m^{2}$. There is thus another interpretation where we use $E_{N}$ and the transformation $\mathrm{v} \rightarrow \phi \mathrm{c}$ and $\mathrm{m} \rightarrow \phi^{3} \mathrm{~m}$ for ordinary energy for quantum particle and $\mathrm{m} \rightarrow 5 \mathrm{~m}$ for dark energy of quantum wave. Finally to find the total energy of Einstein we use $\mathrm{m} \rightarrow\left(5+\phi^{3}\right) \mathrm{m}$ and $\mathrm{v} \rightarrow \phi \mathrm{c}$ where $5+\phi^{3}$ is the dimension of a fractal Kaluza-Klein spacetime [5] for which $\mathrm{D}$ is not 5 but $5+\phi^{3}$.
}

Chart No. 1. The Kaluza-Klein energy of the quantum particle and the dark energy of the quantum wave. 
Dark Energy, Ordinary Energy and Einstein Energy in a nutshell

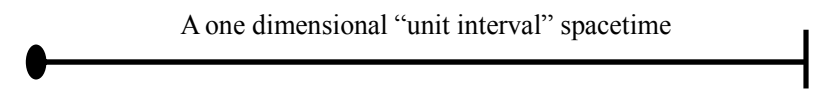

$\mathrm{D}($ Topological $)=\mathrm{D}($ Hausdorff $)=1$

We construct a Cantor set from the one dimensional clopen i.e. closed and open spacetime interval

...........

Uncountably infinitely many points

$\mathrm{D}($ Topological $)=(\mathrm{D}($ Point $)=0$

$\mathrm{D}($ Hausdorff $)=\frac{\sqrt{5}-1}{2}=\phi$
The dimension of the random empty gaps left over from the construction of the Cantor set is acountablely infinitely many gaps given by

$\mathrm{D}($ Topological $)=\mathrm{D}($ Empty set $)=-1$

$\mathrm{D}($ Hausdorff $)=\mathrm{D}($ unit interval $)-(D=\phi)$

$$
\begin{aligned}
& =1-\phi \\
& =\phi^{2}
\end{aligned}
$$

Lifting $\phi^{2}$ additively (union operation) to 5 dimensional Kaluza-Klein spacetime we find a Hausdorff density orvolume $\mathrm{vol}_{H}=5 \phi^{2}$ dimensional Kaluza-Klein spacetime, we find a geometrical density or Hausdorff volume $\operatorname{vol}_{H}=(\phi)^{5}$

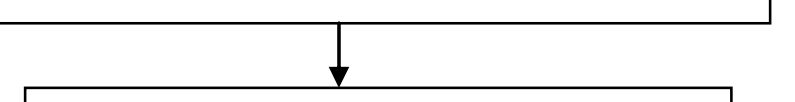

Newton Kinetic energy $E=\frac{1}{2} m v^{2}$ for $V_{O} l_{H}=\phi^{5}$ is thus:

$$
\begin{aligned}
E_{1} & =\left(\phi^{5} / 2\right) m(v \rightarrow c)^{2} \\
& \square m c^{2} / 22 \\
= & \text { Energy of the quantum particle } \\
= & \text { Ordinary Energy }
\end{aligned}
$$
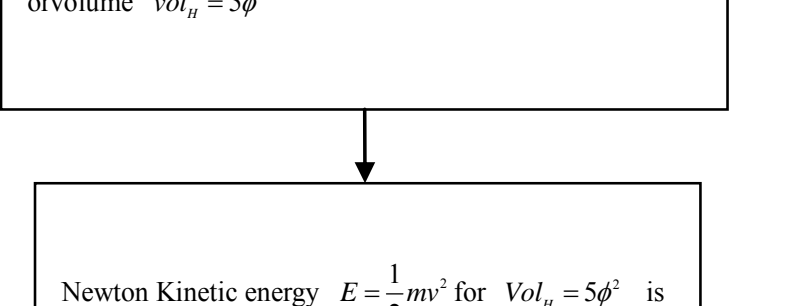
thus:

$$
\begin{gathered}
E_{2}=\left(\phi^{5} / 2\right) m(v \rightarrow c)^{2} \\
\square\left(m c^{2} / 22\right)(21)
\end{gathered}
$$

$=$ Energy of the quantum wave

$=$ Dark Energy

$$
\begin{aligned}
& E=\frac{1}{2} m v^{2} \\
& \text { ntum wave }
\end{aligned}
$$

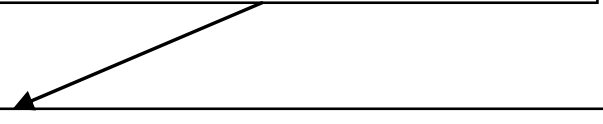

Adding $E_{1}$ of the quantum particle to $E_{2}$ of the quantum wave we find

$$
E=\left(\phi^{5}+\phi^{2}\right)\left(\frac{1}{2}\right) m c^{2}=m c^{2}=E(\text { Einstein })
$$

Chart No. 2. Einstein energy as the sum of the ordinary energy of the zero set particle and the dark energy of the empty set wave in five dimensions. 
Comparative analysis of ordinary quantum particle positive energy and dark quantum wave negative energy

The multiplicative Hausdorff volume vol(D(0)) of the zero set given by $\mathrm{D}(0)=(0 ; \phi)$ which represents the quantum particle is

$\operatorname{vol}(D(0))=(\phi)^{D_{s}}$

Taking $D_{s}=5$ for a Kaluza-Klein spacetime one finds $\operatorname{vol}(D(0))=\phi^{5}$. Note that: $\phi^{5} \quad$ where is the Hardy probability for quantum entanglement of two particles $\phi=(\sqrt{5}-1) / 2$.
The dual to the Hausdorff volume of the zero set is the additive pseudo Hausdorff volume of the empty set: $\operatorname{vol}(D(-1))=\left(-1, \phi^{2}\right) \quad$ which represents the quantum wave. Consequently $\operatorname{vol}(D(-1))$ in five dimensions of Kaluza-Klein space is $\operatorname{vol}(D(-1))=(5)\left(\phi^{2}\right)$. Note that the empty set is the cobordism of the zero set. That means it is the surface of the quantum particle is the quantum wave. for there more $\left(5 \phi^{2}\right)$ could be given a negative sign because of the $(-1)$ component of $D(-1)$ so that strictly speaking $\operatorname{vol}(D(-1))$ is a negative volume in the measure theoretical meaning attached to a negative Menger-Urysohn deductive dimensional system.
Newton kinetic energy $E=\frac{1}{2} m v^{2}$ for $\operatorname{vol}(\mathrm{D}(0))$

and $\mathrm{v} \rightarrow \mathrm{c}$ where $\mathrm{c}$ is the speed of light becomes

$$
\begin{aligned}
E_{1} & =\operatorname{vol}\left(D_{0}\right)\left(\frac{1}{2} m c^{2}\right) \\
& =\left(\phi^{5} / 2\right) m c^{2} \\
& \square m c^{2} / 22 \\
& =\text { ordinary Energy }
\end{aligned}
$$

which is the energy of a quantum particle

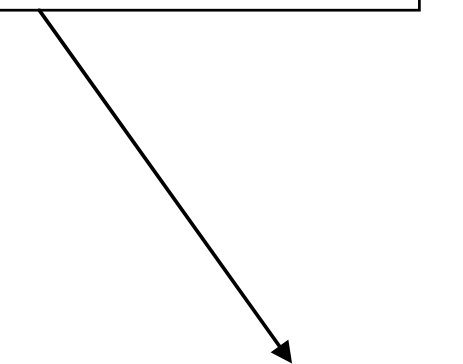

Newton Kinetic energy $E=\frac{1}{2} m v^{2}$ for $\operatorname{vol}(D(-1))$ and $v \rightarrow c$ becomes:

$$
\begin{aligned}
E_{2} & =\operatorname{vol}(D(-1))\left(\frac{1}{2} m c^{2}\right) \\
& =\left(5 \phi^{5} / 2\right)\left(m c^{2}\right) \\
& \square\left(m c^{2}\right)(21 / 22) \\
& =\text { Dark Energy }
\end{aligned}
$$

which is equal to the negative energy

$(-1)\left(\mathrm{mc}^{2}\right)(21 / 22)$ producing negative gravity which is a halo energy caused by the quantum wave

Chart No. 3. The energy corresponding to the wave-particle duality and the corresponding empty set-zero set duality in $D=5$ Kaluza-Klein spacetime. 


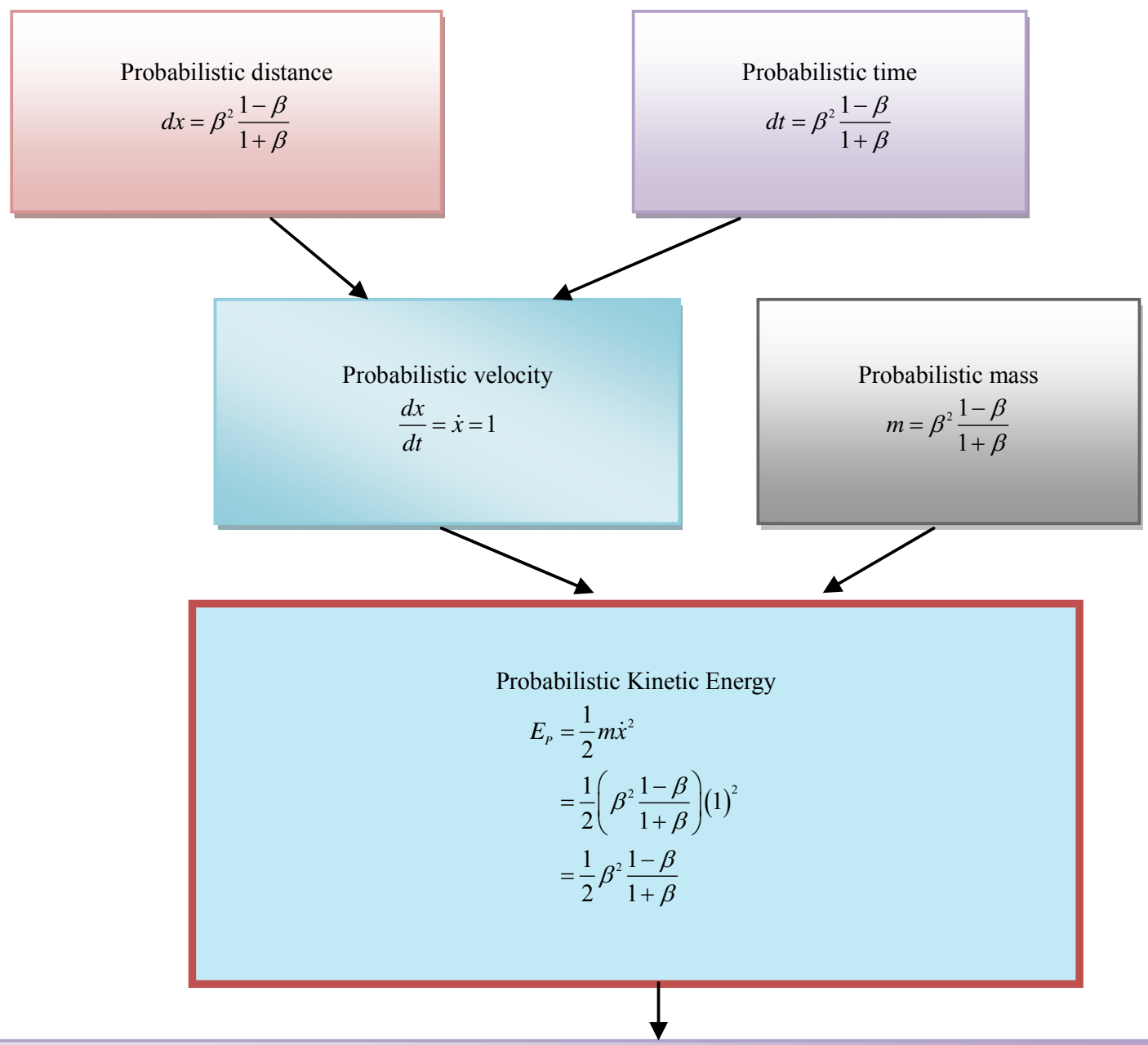

Either setting $\beta=\phi=(\sqrt{5}-1) / 2$ or equating $\mathrm{E}_{\mathrm{L}}$ to $\mathrm{E}_{\mathrm{P}}$ and finding that $\beta=\phi$. That way the probabilistic $\mathrm{E}_{\mathrm{P}}$ is simply equal to $\mathrm{E}_{\mathrm{L}}$ of the light cone,so that

$$
E_{P}=E_{L}=P(H)=\phi^{5} / 2
$$

The final Result is:

$$
\begin{aligned}
E_{Q R} & =E_{P}\left(m c^{2}\right) \\
& =\left(\phi^{5} / 2\right)\left(m c^{2}\right)
\end{aligned}
$$

\section{Important note: $E_{p}$ here is a probabilistic E and should not be confused with Planck energy Ep.}

Chart No. 4. Flow chart for probabilistic strategy to derive E-quantum relativity using Hardy's quantum entanglement probability method for $P(H)=\left(\phi^{5} / 2\right)$. 


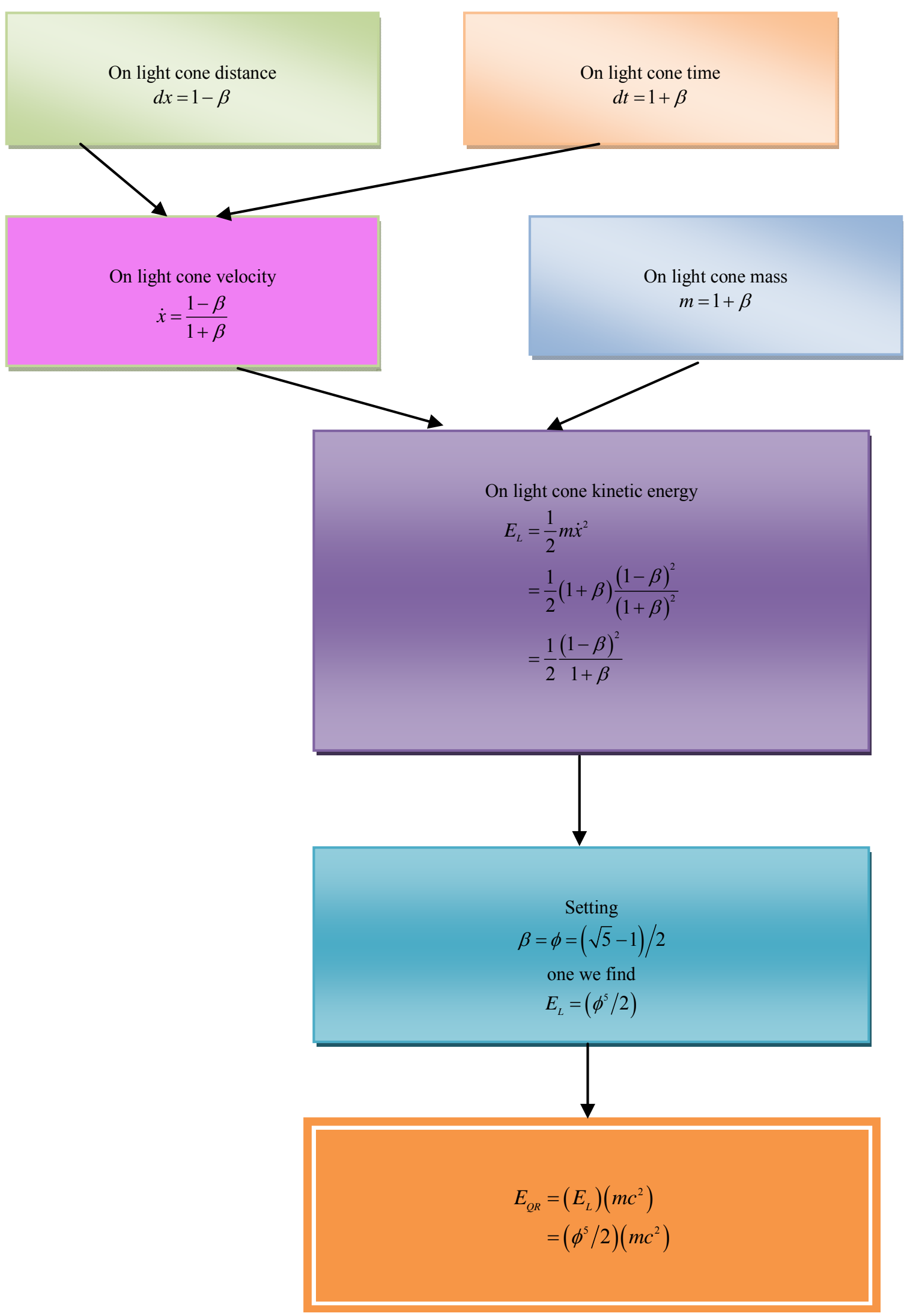

Chart No. 5. Flow chart for the on light cone strategy to derive E-quantum relativity as in the light cone quantization of super string theory. 


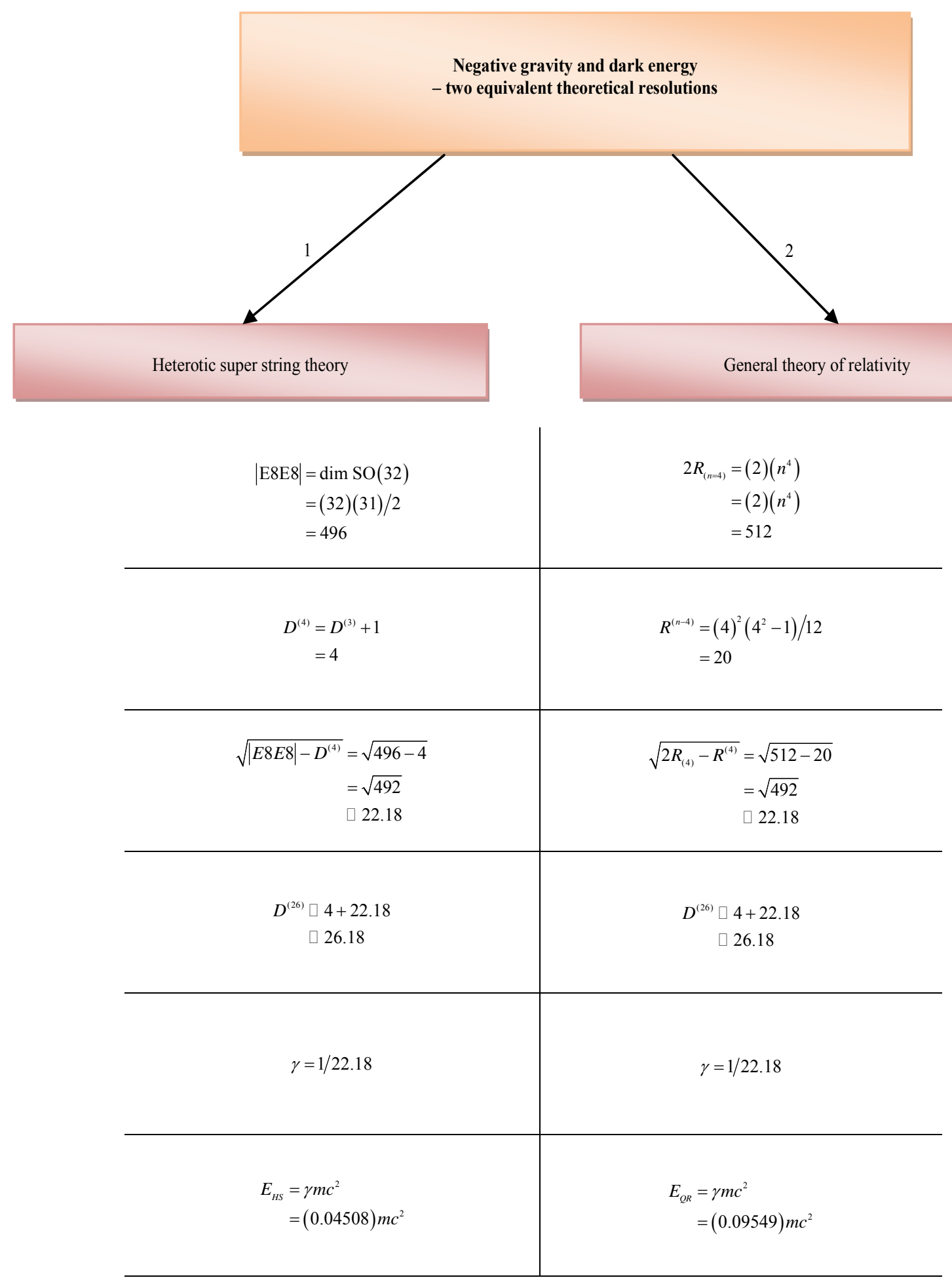

WMAP and supernova data confirmed that $95.4 \%$ of the energy of the cosmos is missing or at least could not be detected by any of the known methods. In addition accurate measurement shows that the expansion of the universe is accelerating. The above result $\quad\left(\gamma=1 / 22.18\right.$ and $\left.\frac{E(\text { Dark })}{m c^{2}}=95.4 \%\right)$ completely agrees with cosmic measurements and data.

Chart No. 6. The dark energy of the quantum wave as deduced from the ordinary energy of the quantum particle using general relativity and Heterotic superstrings. 


\section{Geometrical interpretation of the energy equation of quantum relativity} $E_{Q R} \square m c^{2} / 22$ and Hardy's quantum entanglement probability

\section{$\underline{\text { The E8E8 string's triangle }}$}

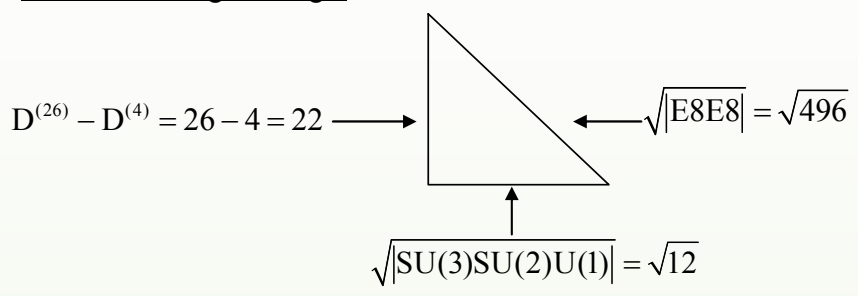

Here E8 is the exceptional Lie Symmetry group of rank 8 and SU(3)SU(2)SU(1) are the combined Lie groups of the standard model Symmetry Group

The transfinite E8E8 string's triangle and its quantum entanglement interpretation

$$
\begin{aligned}
\mathrm{D}^{(26)}-\mathrm{D}^{(4)} & =(26+\mathrm{k})-4 \\
& =22+\mathrm{k} \longrightarrow \\
& =22.18033929 \\
& \sqrt{\mathrm{D}^{(4)}}=\sqrt{4}=2
\end{aligned}
$$

The triangular area $A_{c}=\left(\frac{1}{2}\right)(22+k)(2)=2 / \phi^{5}$ where $\phi=(\sqrt{5}-1) / 2, k=2 \phi^{5}$ and $\phi^{5}=\frac{1}{22+k}$.

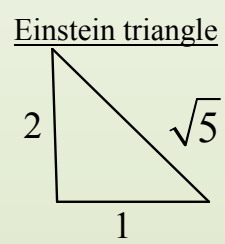

The area $A=\gamma($ Einstein $)=\frac{1}{2}(2)(1)=1$ where $E=\gamma m c^{2}=m c^{2}, \sqrt{5}=2+\phi^{3}, \sqrt{5}+1=3+\phi^{3}, \sqrt{5}+2=4+\phi^{3}$ and $\sqrt{2}+1+2=5+\phi^{3}$

\section{$\underline{\text { Conclusion }}$}

The Lorentz factor of $E_{Q R}$ is $\gamma_{Q R}=1 / A_{c}=\left(\phi^{5} / 2\right)=\frac{1}{22+k} \square \frac{1}{22} m c^{2}$ and consequently we have

$$
\begin{aligned}
& E_{Q R}=\frac{E(\text { Einstein })}{\text { Area }\left(A_{c}\right)}=\frac{m c^{2}}{22+k} \square m c^{2} / 22 \\
& \text { i.e. } \gamma_{Q R}=\frac{A(\text { Einstein })}{A_{c}}=\frac{1}{22+k} \square \frac{1}{22} \text {. Finally Hardy's quantum probability of entanglement } \\
& \text { is } P(H)=(2)\left(\frac{1}{A_{c}}\right)=\phi^{5}
\end{aligned}
$$

Chart No. 7. Geometrical interpretation of the ordinary energy of a quantum particle and the existence of minimal areas. 